\title{
Anti-algal coating formulation with silica-titania core-shell nanoparticles for exterior architectural application
}

\author{
Arpita Bhattacharya $^{1 *}$, Surbhi Sinha ${ }^{2}$ and Subhasha Nigam ${ }^{2}$ \\ ${ }^{1}$ Amity Institute of Nanotechnology, Amity University, Sec-125, Noida, U.P., India \\ ${ }^{2}$ Amity Institute of Biotechnology, Amity University, Sec-125, Noida, U.P., India \\ *Email: abhattacharya@amity.edu
}

Algal growth on emulsion paint film applied on exterior architectural work is a common problem. Usually paints contain some protective colloids, which helps algal growth. Various anti-algal agents are used in this type of paint formulation. But these are toxic chemicals which can harm environment as well as human beings. Certain nanoparticles like silver nanoparticles [1], Zinc oxide nanoparticles, nano-titania have been tried as anti-algal agent.

In this study, we tried silica-titania core-shell nanoparticles as anti-algal agent. Core-shell nanoparticles exhibit novel properties, which are different to those of core and shell materials individually. In silica/titania core -shell structure core of nano-silica can provide good mechanical and anti-scratch properties whereas nano-titania coating will provide anti-microbial and self cleaning properties [2]. Activity of titania increases as the thickness decreases which is possible in core-shell structure where silica nano spheres host as the core material and titania shell thickness can be tailor-made on it. The anatase form of $\mathrm{TiO} 2$ is more suitable for photo catalysis [3].

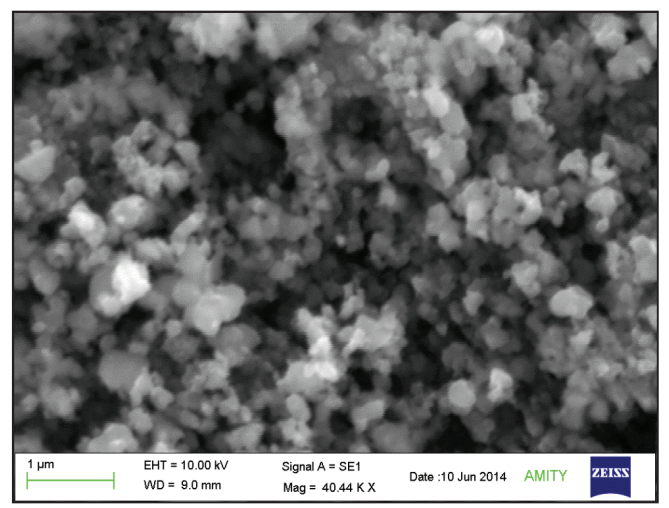

Figure 1 : SEM image of titania coated silica

Strong oxidation power eliminates odour causing bacteria and easily growing microorganisms. In this present work $\mathrm{TiO} 2$ and $\mathrm{SiO} 2 / \mathrm{TiO} 2$ core -shell nanoparticles were synthesized and characterized through UVVIS, DLS, XRD, SEM and FTIR. The SEM of $\mathrm{SiO} 2-\mathrm{TiO} 2$ nanoparticles is shown in Figure 1

The prepared particles were used in acrylic emulsion and the resultant formulations were applied on ceramic tiles. These were put in algal growth media and tested for inhibition effect of the nanoparticles on algal growth. Both titania and core-shell showed complete killing of bluegreen as well as green algae species in the culture media which is shown in Figure 2.

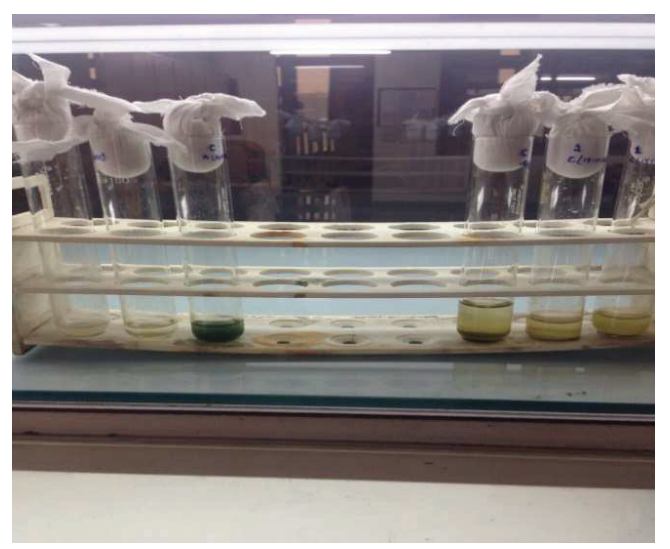

Figure 2: Titania and silica-titania nanoparticles in culture tubes containing blue-green (left) and green algae (right) species

So it is expected that with this core-shell structure, an anti-algal coating formulation could be developed specially for white and light coloured coating formulation.

\section{References}

1. L.W. Menno \& L.H. Koole, Polymers, 3 (2011), 340 .

2. J.W. Molling et al., Nanotechnology Sci. Appl., 7(2014), 97.

3. V.K. Yenomireddy et al., J. Food Sci, 80(8) (2015) 1903 